\title{
BMJ Open Quality Reducing severe hypoglycaemia in hospitalised patients with diabetes: Early outcomes of standardised reporting and management
}

\author{
Katherine A Araque, ${ }^{1}$ Deepak K Kadayakkara, ${ }^{1}$ Nino Gigauri, ${ }^{1}$ Diane Sheehan, ${ }^{2}$ \\ Sachin Majumdar, ${ }^{3}$ Gregory Buller, ${ }^{1}$ Clare A Flannery ${ }^{4}$
}

To cite: Araque KA,

Kadayakkara DK, Gigauri N, et al. Reducing severe hypoglycaemia in hospitalised patients with diabetes: Early outcomes of standardised reporting and management.BMJ Open Quality 2018;7:e000120. doi:10.1136/ bmjoq-2017-000120

Received 15 May 2017 Revised 28 March 2018 Accepted 29 March 2018
Check for updates

${ }^{1}$ Internal Medicine Department, Yale New Haven Health Bridgeport Hospital, Bridgeport, Connecticut, USA

${ }^{2}$ Endocrinology Department, Yale New Haven Health Bridgeport Hospital, Bridgeport, Connecticut, USA

${ }^{3}$ Endocrinology Department, Yale New Haven Health Bridgeport Hospital, Bridgeport, Connecticut, USA

${ }^{4}$ Section of Endocrinology, Internal Medicine Department, Yale School of Medicine, New Haven, Connecticut, USA

Correspondence to Dr Gregory Buller; Gregory.buller@bpthosp.org

\section{ABSTRACT}

Background Severe hypoglycaemic events (HGEs) in hospitalised patients are associated with poor outcomes and prolonged hospitalization. Systematic, coordinated care is required for acute management and prevention of HGEs; however, studies evaluating quality control efforts are scarce.

Objective To investigate the effectiveness of systembased interventions to improve management response to HGEs.

Methods System-based interventions were designed and implemented following a root cause analysis of HGE in adult patients with diabetes from two general medical wards with the highest incidence of HGE. Interventions included electronic medical record programming for a standardised order set for basal-bolus insulin regimen and hypoglycemia protocol, automated dextrose order, automated MD notification, and recommendation for endocrine consultation after two critical HGEs. The Pyxis MedStation was programmed to alert nurses to recheck blood glucose $15 \mathrm{~min}$ after the treatment. A card with the HGE management protocol was attached to each provider's ID badge and educational seminars were given to all providers.

Main outcomes and measures Primary outcomes were to evaluate median time from HGE (glucose $<50 \mathrm{mg}$ / $\mathrm{dL}$ ) to euglycemia (>100 $\mathrm{mg} / \mathrm{dL}$ ), and time from HGE to follow-up finger-stick (FS) testing preintervention and postintervention. Secondary outcomes were cumulative incidence of HGEs, recurrent hypoglycemia, rate of physician notification and use of standardised treatments among adults with diabetes on the two general medical wards.

Results Among hospitalised adults with diabetes and HGE, median time from HGE to euglycemia declined from $225 \pm 46$ min preintervention to $87 \pm 26$ min postintervention $(p=0.03)$. Median time from HGE to next FS testing also declined ( $76 \pm 14$ min to $28 \pm 10 \mathrm{~min}, \mathrm{p}<0.001$ ). Standardised treatment administration for HGE improved significantly from $34 \%(12 / 35)$ to $97 \%$ (36/37); physician notification rate improved significantly from $51 \%(18 / 35)$ to $78 \%(29 / 37)$. Among hospitalised adults with diabetes, incidence of HGE decreased from 12\% (35/295) over 3 months (preintervention period) to $6 \%(37 / 610)$ over 6 months (postintervention period) $(p<0.001)$, while recurrent HGE did not show significant differences $(37 \%$ (13/35) to $24 \%(9 / 37), p=0.09)$.
Conclusions System-based interventions had a clinically important impact on decreasing time from HGE to euglycemia and to next FS testing. This hypoglycemia bundle of care may be applied and tested in other community hospitals to improve patient safety.

\section{INTRODUCTION}

Severe hypoglycaemia (serum glucose level less than $50 \mathrm{mg} / \mathrm{dL}$ ) in hospitalised patients is an adverse consequence of intensive glucose management. Tighter blood glucose before and after eating have been advocated over the last decade to reduce rates of infection and poor cardiovascular outcomes, ${ }^{1-4}$ but a tight glycaemic target can increase hypoglycaemia as shown in the intensive treatment group of the ACCORD trial (The Action to Control Cardiovascular Risk in Type 2 Diabetes) where a significant number of asymptomatic hypoglycaemic event (HGEs) were identified. ${ }^{5}$ HGEs during hospitalisation are associated with poor outcomes including autonomic failure, arrhythmias, cognitive decline ${ }^{6-9}$ and increased morbidity and mortality within 1 year of discharge. ${ }^{10}$ Inpatient hypoglycaemia in patients with diabetes leads to longer lengths of stay, and greater likelihood for discharge to a skilled nursing facility. The economic burden of critical hypoglycaemia is well known and includes increased rates of readmission and increased cost of the hospitalisation up to $50 \%$ in a given HGE. ${ }^{11}$ Rapid recovery from HGE to euglycaemia is important because the duration of hypoglycaemia is linked with adverse outcomes such as neuronal damage. ${ }^{12}$ Longer duration and repetitive episodes of hypoglycaemia activate glucose counter-regulatory hormones, which can lead to a diminished autonomic response and unawareness hypoglycaemia. ${ }^{13} 14$

Multiple professional societies, including the American Diabetes Association (ADA) and the Endocrine Society, recommend that 
all hypoglycaemic episodes be evaluated for a root cause. Hospitalised patients are at risk for hypoglycaemia due to fluctuating illness, unpredictable periods of fasting, side effects of medications and altered ability to report symptoms. Thus, clinical care needs to be tightly coordinated among nursing and technical staff, medical teams, pharmacy, nutrition and administrative staff. A standardised approach and management are generally recommended to maintain glycaemic targets. ${ }^{15}$ However, the prevalence of inpatient HGE is generally unknown, and system-based methods to prevent HGE and improve management are scarce in the literature. Prior retrospective studies have shown a wide variation of diabetes care measurements in academic medical centres, such as HbA1c assessment, timely admission laboratory glucose measurement and glucose goals in different levels of care like intensive care unit (ICU) versus intermediate care versus ward with overall suboptimal glucose control despite frequent insulin use. ${ }^{16}$ Maynard et $a l^{17}$ demonstrated how a hypoglycaemia bundle reduces glucose excursions and HGEs in non-ICU setting.

We performed a root cause analysis of severe HGE in hospitalised patients with diabetes to identify factors associated with a prolonged time to recovery from the event. Subsequently, we used a multidisciplinary approach to design system-based interventions to address the root factors. This study aims to assess the effectiveness of thishypoglycaemia bundle in reducing the time to recovery from an HGE and improving the management response in a single hospital setting.

\section{METHODS}

\section{Study design and participants}

Systematic review of critical HGEs: An initial review of HGE at the Yale New Haven Health Bridgeport Hospital, a 393-bed third-level general medical and surgical teaching hospital in Connecticut was collected. We identified two inpatient general internal medicine wards with the highest incidence of HGE, defined as a serum glucose $<50 \mathrm{mg} / \mathrm{dL}$ per venous serum glucose or finger stick (FS). Recurrent HGE was defined as glucose $<50 \mathrm{mg} / \mathrm{dL}$ more than once in a given patient during a single hospitalisation. Each HGE which occurred in either ward over a 3-month period was assessed through a detailed review of electronic medical records (EMRs), as part of a quality control protocol exempted from institutional review board approval. We included HGEs occurring in patients with a diagnosis of diabetes type 1 or type 2 (primary or secondary as per the International Classification of diseases ICD-9/ICD10 coding system) and who consented to glucose monitoring by FS or basic chemistry panel as standard of care. Exclusion criteria included the absence of diabetes diagnosis and glucometer performance errors.

Data collection occurred preintervention over 3 months and included patient demographics, body mass index (BMI), serum albumin, renal function, antihyperglycaemic therapy (insulin, other injectable or oral
Table 1 Hypoglycaemia bundle.

\begin{tabular}{ll}
$\begin{array}{l}\text { Hypoglycaemia bundle-interventions to improve } \\
\text { treatment of HGE }\end{array}$ \\
\hline $1 \quad \begin{array}{l}\text { Standardisation of treatment (glucose gel, intravenous } \\
\text { dextrose or intramuscular glucagon) }\end{array}$ \\
$2 \quad \begin{array}{l}\text { Dextrose administration by nursing staff without } \\
\text { requirement for physician orders and Pyxis } \\
\text { MedStation alert to check follow-up FS after } \\
\text { treatment }\end{array}$ \\
$\begin{array}{l}\text { EMR order set for insulin regimen: basal and bolus } \\
\text { correction and automated hypoglycaemia order set }\end{array}$ \\
$\begin{array}{l}\text { Automated physician notification immediately after } \\
\text { HGE to reassess risks }\end{array}$ \\
$\begin{array}{l}\text { After two HGEs, automated best practice advisor on } \\
\text { EMR with recommendation for endocrine consultation } \\
6\end{array}$ & $\begin{array}{l}\text { Standardised hypoglycaemia management protocol: } \\
\text { laminated card attached to staff ID badge }\end{array}$ \\
7 & $\begin{array}{l}\text { Education of nursing staff and inpatient medical } \\
\text { teams }\end{array}$
\end{tabular}

EMR, electronic medical record; FS, finger stick; HGE, hypoglycaemic event.

medication) administered prior to HGE. Data following the HGE included time to next FS, treatment of HGE, notification to MD and if applicable, resulting changes in insulin dose. We classified risk factors contributing to slow response to events into four major categories: equipment and supplies, procedure (hospital policy), communication and staff.

Data were similarly collected during the postintervention interval for 6 months. The times were defined based on documented times from the EMR. All the data collected were registered on a database for further statistical analysis.

Establishment of a hypoglycaemia committee: The committee was responsible for the design, implementation and evaluation of a standardised multidisciplinary approach for management of HGE. The committee consisted of physicians, nurses, nurse assistants, pharmacists, dietitians and administrators which met on a weekly basis. The committee created an initiative called the 'hypoglycemia bundle of care' which includes systembased interventions (table 1) to address systemic error and delays affecting recovery times from HGEs, as identified by the root cause analysis (figure 1). The bundle of care was implemented after the approval of all members of the committee.

\section{System-based interventions \\ Equipment and supplies}

The committee identified that glucometer readings were transmitted automatically to the medical record system without creating an alert. Thus there was no clear responsibility regarding which staff member was supposed to follow-up the reading, leading to delays in recognition and treatment. Furthermore, there was no standardised 


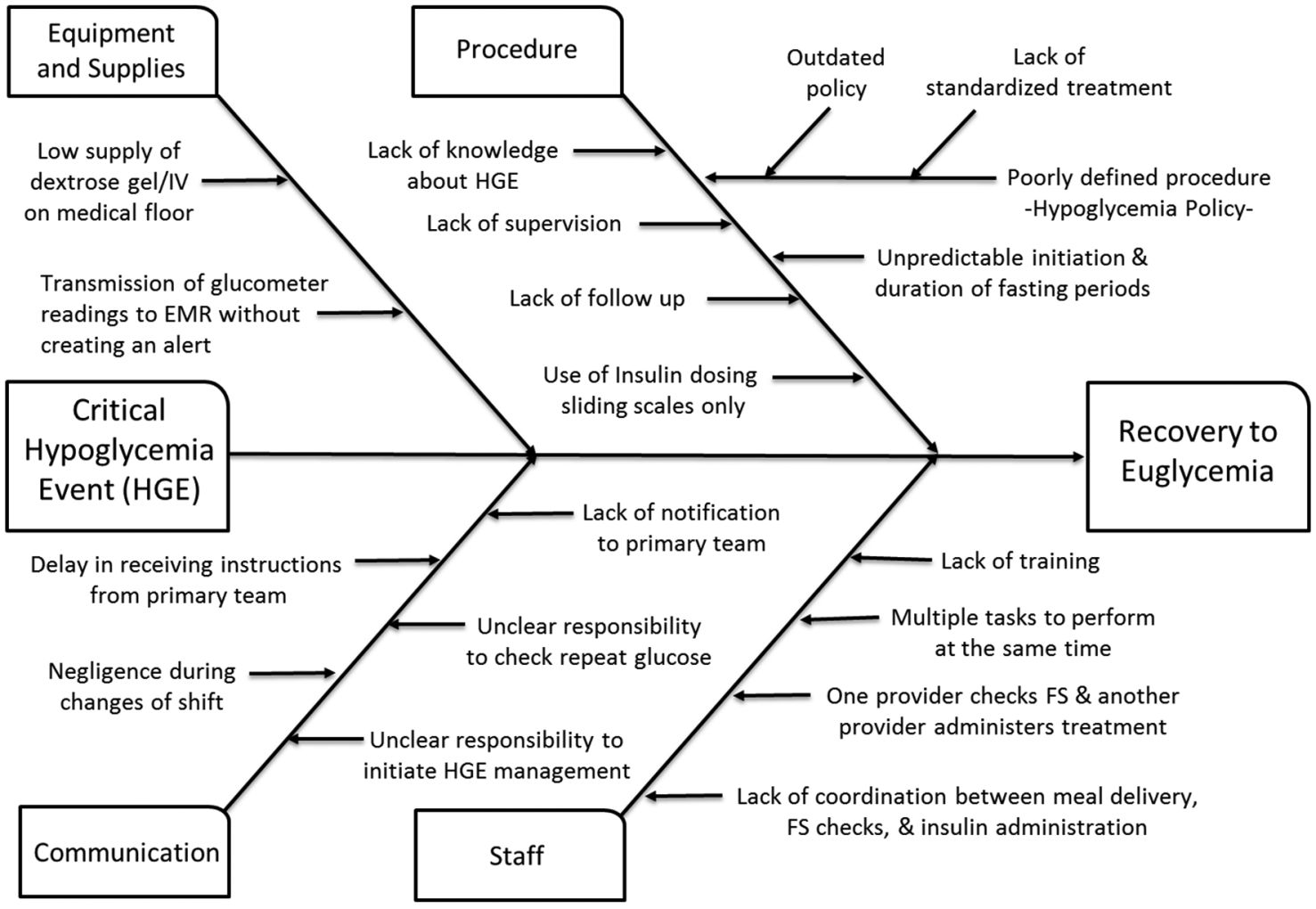

Figure 1 Root cause analysis of hypoglycaemic events (HGE). EMR, electronic medical record; FS, finger stick; IV, intravenous.

policy regarding follow-up FS after treatment administration. All nursing staff received education from the diabetes educator regarding the importance of following up automatic transmission of FS results to the EMR. The committee also recognised that there were limited supplies of dextrose gel/intravenous available to the nursing staff in the medical wards. Thus, pharmacy increased delivery of dextrose supplies for use in the medical wards and an automatic alert was created once the supplies were used.

\section{Procedure and hospital policy}

Outdated policy limited the therapeutic intervention to carbohydrate administration and did not have instructions on follow-up care. The committee created a standardised treatment plan with intravenous glucose, dextrose gel or intramuscular glucagon rather than providing carbohydrate-based 'snacks'. A laminated badge with an algorithmic response to HGE and non-critical HGE was given to all healthcare providers and worn around their neck next to their identification (ID) card (figure 2). The badge included reminders to notify the primary team to evaluate, identify risk factors and modify insulin dosages. For the first time, nurses were authorized to administer glucose gel or intravenous dextrose, as part of the hypoglycaemic order set included in any insulin order by the physician on admission. They were also able to activate the dextrose emergently during an HGE. The new policy included a standardised FS after $15 \mathrm{~min}$ of treatment and required an $\mathrm{FS}>100 \mathrm{mg} / \mathrm{dL}$ to define recovery; to improve adherence to FS checking after treatment, a new alert in the Pyxis MedStation was created to recheck blood glucose 15 min after dextrose or glucagon administration. This alert required an acknowledgement. The glucometer continues to transmit data automatically, and the responsibility for responding to the alert was designated to the nurse assigned to each patient. Nurses can administer dextrose immediately because the orders for dextrose are placed automatically on admission so there is no time delay waiting for a physician to place the order.

For the first time, a standardised order set for basal-bolus insulin regimen ${ }^{18-20}$ designed by health network endocrinologists and based on the ADA standard of care was established in the EMR after committee approval. This regimen included an automated order set for a standardised management of hypoglycaemia. We created a 'best practice advisor alert' on our medical record system which automatically recommends an endocrinology consultation after two consecutive HGEs for a given patient.

\section{Staff and communication}

To address human errors leading to prolonged time from HGE to recovery, we initiated an educational programme provided by a diabetes educator to healthcare providers, including nurses, technicians and primary medical teams. The educational programme focused on the situation, background, assessment and recommendations technique which trained the staff members about the new policy initiatives.

\section{Outcomes}

The primary outcomes of our study were time to recovery defined as median time from HGE to euglycaemia 

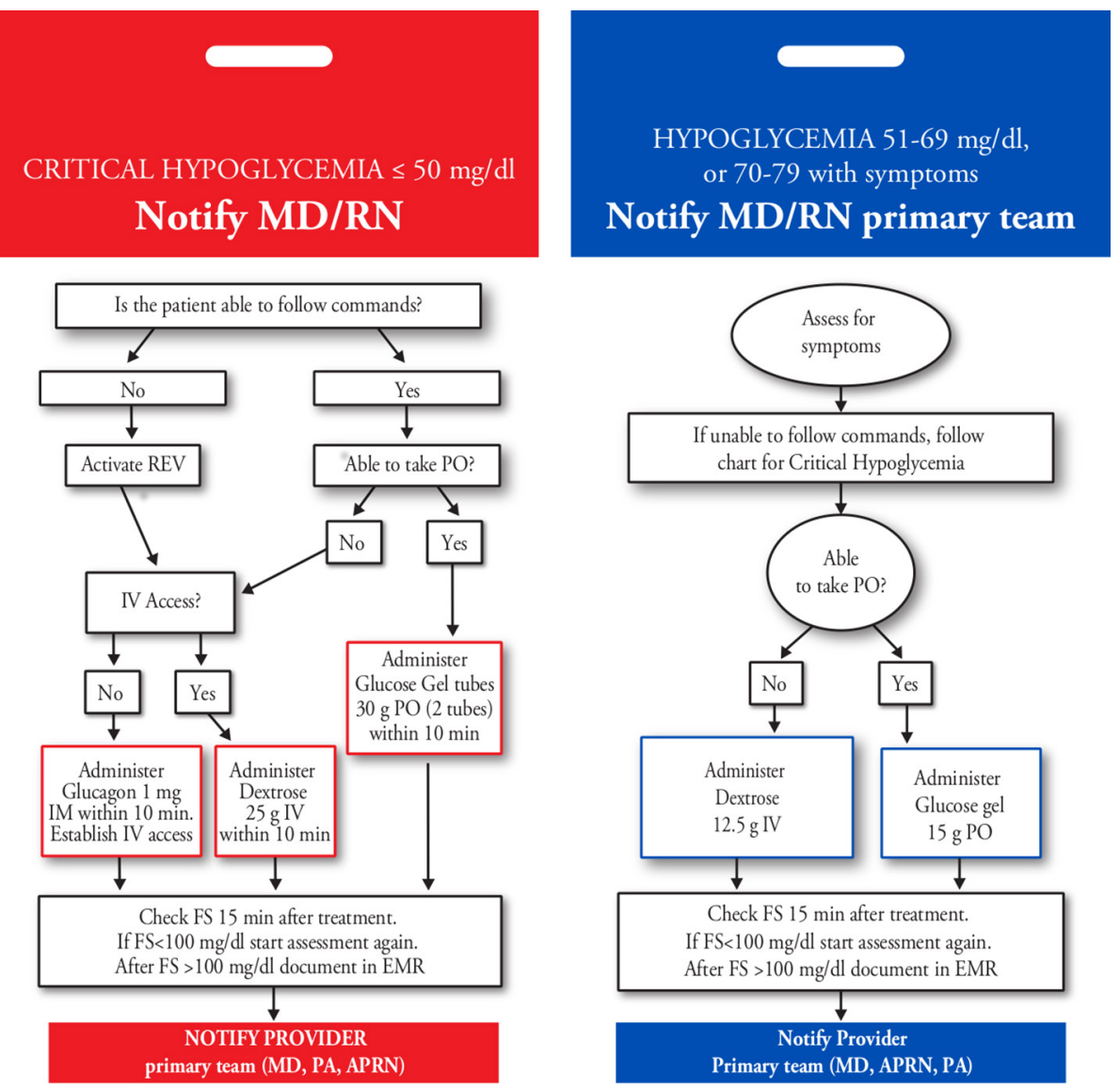

Figure 2 Standardised management protocol for (A) critical hypoglycaemia ( $<50 \mathrm{mg} / \mathrm{dL})$ and (B) hypoglycaemia $50-69 \mathrm{mg} /$ $\mathrm{dL}$ or $70-79 \mathrm{mg} / \mathrm{dL}$ with symptoms. Protocol written as a laminated card attached to staff identification badge. EMR, electronic medical record; FS, finger stick; IV, intravenous; PO, orally; REV, rapid emergency evaluation team; MD, medical doctor; RN, registered nurse; APRN, advanced practiced registered nurse; PA, physician assistant.

(BG $>100 \mathrm{mg} / \mathrm{dL}$ ) and time to next FS measurement following treatment of HGE. The secondary outcomes of the study were cumulative incidence of HGE, physician notification rate, administration of standardised treatment and recurrent HGE in a single hospitalisation. Cumulative incidence was measured for the observed 3-month period pre-intervention and 6-month period post-intervention.

\section{Statistics}

The data was summarised and the primary outcomes, that is, median time to recovery from hypoglycaemia to euglycaemia and time to next FS measurements were calculated. The histogram analysis showed that the data was not normally distributed. The statistical significance of the data pre-intervention and post-intervention was tested by the Mann-Whitney $\mathrm{U}$ test. The secondary outcomes that consisted of categorical values were tabulated in contingency tables and $\chi^{2}$ test was used to test for statistical significance. The data was analysed using Graphpad Prism V.6 (La Jolla, California, USA).

\section{RESULTS}

We identified 49 patients who had 72 critical HGEs during the duration of our study (table 2). The at-risk population was mainly those with type 2 diabetes (92\% and $77 \%$, respectively for each period), with a mean age of 60 years, and no gender predominance. A majority $(64 \%)$ had some degree of renal injury (eGFR $<60 \mathrm{~mL} / \mathrm{min}$ ). Among patients in the pre-intervention group ( $\mathrm{n}=22), 73 \%$ received insulin alone, $5 \%$ received oral agents alone and 22\% received combined therapy. In the post-intervention group $(n=27)$, $62 \%$ received insulin alone, $14 \%$ received oral treatment alone and $28 \%$ received combined treatment.

\begin{tabular}{lll}
\hline Table 2 Patient demographics. \\
\hline \multicolumn{3}{l}{ Characteristics of patients with HGE } \\
\hline & $\begin{array}{l}\text { Pre-intervention } \\
\text { (3months) }\end{array}$ & $\begin{array}{l}\text { Post-intervention } \\
\text { (6 months) }\end{array}$ \\
\hline Number of patients & 22 & 27 \\
Number of HGEs & 35 & 37 \\
\hline Type 2DM, n (\%) & $20(92 \%)$ & $21(77 \%)$ \\
Mean age $\pm S E M$ & $60 \pm 12$ & $68 \pm 3$ \\
\hline M:F ratio & $50: 50$ & $43: 57$ \\
BMl>30, n (\%) & $9(41 \%)$ & $5(19 \%)$ \\
GFR<60, n (\%) & $14(64 \%)$ & $18(67 \%)$ \\
\hline
\end{tabular}

BMI, body mass index; HGE, severe hypoglycaemic event; GFR, Glomerular filtration rate. 
A

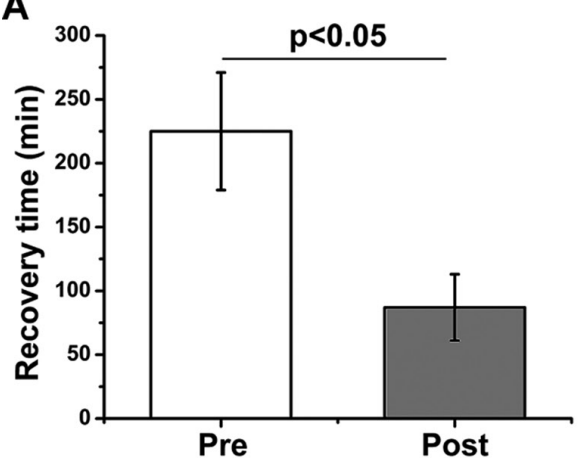

C
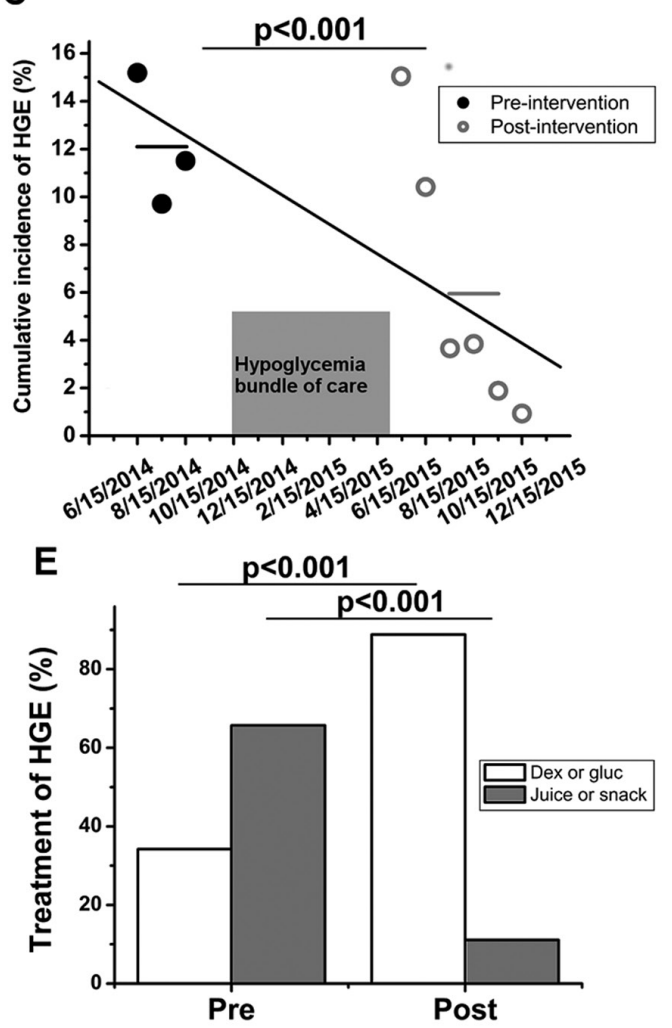

B

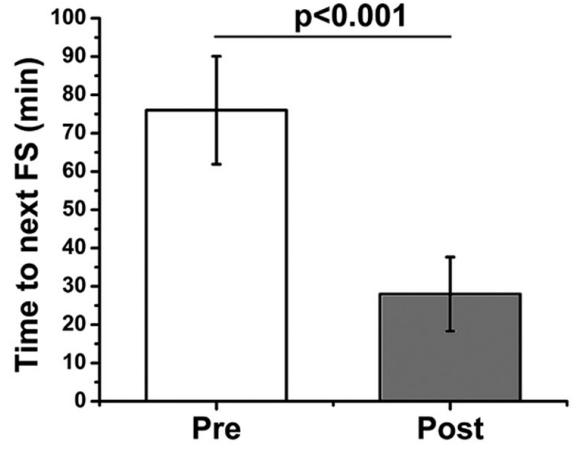

D

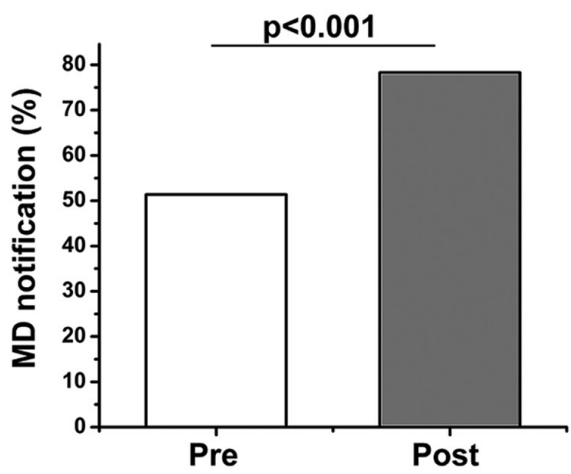

$\mathbf{F}$

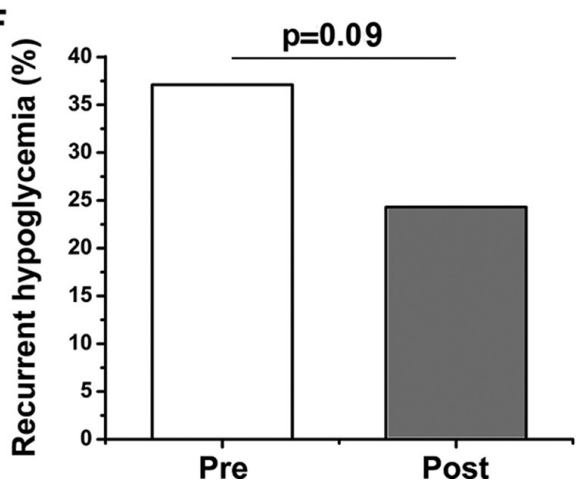

Figure 3 Primary outcomes: (A) median time to recovery from hypoglycaemic event (HGE) to euglycaemia and (B) median time from HGE to next finger stick (FS) glucose measurement pre-intervention and post-intervention. Secondary outcomes: (C) cumulative incidence of HGE. The grey shadow indicates the time period when the hypoglycaemia bundle of care was planned, (D) physician notification rate, $(E)$ treatment rate and $(F)$ recurrent hypoglycaemia pre-intervention and post-intervention. Error bars show SEM.

From pre-intervention to post-intervention, the median time from HGE to recovery declined by $138 \mathrm{~min}$ $(61 \%)$, from $225 \pm 46 \mathrm{~min}$ to $87 \pm 26 \mathrm{~min}(\mathrm{p}=0.03)$. Median time from initial HGE to next FS check was also significantly reduced, from $76 \pm 14 \mathrm{~min}$ to $28 \pm 10 \mathrm{~min}(\mathrm{p}<0.001)$ (figure 3,B).

The monthly cumulative incidence of critical HGE progressively decreased post-intervention. Overall, during the study period it reduced from $12 \%$ to $6 \%(\mathrm{p}<0.001)$ (figure 3C). Recurrent HGE was lower in the context of an overall decline in the number of HGEs, $37 \%(13 / 35$ pre-intervention) and $24 \%(9 / 37)$ post-intervention ( $\mathrm{p}=0.09)$ (figure 3F).
Physician notification increased from 51.4\% (18/35) pre-intervention to $78 \%(29 / 37)$ post-intervention $(\mathrm{p}<0.001)$ (figure 3D). Administration of standardised treatment (intravenous dextrose or oral glucose gel) increased from $34 \%(12 / 35)$ to $97 \%(36 / 37) \quad(\mathrm{p}<0.001)$ (figure $3 \mathrm{E})$. Patients who received intravenous dextrose had significantly faster recovery time $(33 \pm 42 \mathrm{~min})$ compared with those who received dextrose gel $(184 \pm 37 \mathrm{~min})(\mathrm{p}=<0.012)$. Of note, a few patients refused dextrose gel due to bad taste and thus continued to received carbohydrate-based snacks.

\section{DISCUSSION}

Severe hypoglycaemia among hospitalised patients is common, poses significant health threats to patients and 
requires systemic interventions to improve outcomes. Our study assessed critical HGEs in hospitalised patients with diabetes to determine factors that prolonged time to recovery from as HGE and to FS follow-up. We created a multidisciplinary hypoglycaemia committee, performed a root cause analysis of HGEs on two inpatient wards with the highest rates of these events, and implemented a hypoglycaemia bundle-of-care intervention. Following the intervention, we found significant improvements in the management of HGE: a decrease in the time from HGE to euglycaemia by $61 \%$ and from HGE to next FS by $63 \%$. We highlight three important aspects in hospital management of critical HGEs: (1) the importance of a root cause analysis in recognising and addressing unique system failures in a given medical setting (2) initiating a hypoglycaemia bundle of care reduces the time to recovery and follow-up FS measurement; (3) standardising the assessment and management of HGEs, improves patient safety and helps to deliver high-quality care. The timing of the intervention in response to hypoglycaemia is paramount for improving patient outcomes. Based on our observations, treatment with intravenous dextrose resulted in faster time to recovery; however further studies are needed to confirm this observation. While the EMR software enables automatic downloading of glucose measurements, it can be a double-edged sword as this requires provider designation for follow-up. The Institute of Medicine report states 'The need for a stronger focus on evidence to ensure that the promise of scientific discovery and technological innovation is efficiently captured to provide the right care for the right patient at the right time'. ${ }^{21}$ By standardising procedures and implementing the hypoglycaemia bundle of care, patients in our two hospital ward units received increased recognition, treatment and follow-up of critical hypoglycaemia in a timely manner. Improvements in communication and easy access to treatment algorithms helped all staff to provide a comprehensive management plan. The root cause analysis model and failure mode and effects analysis can help evaluate systems to redesign and correct processes and human errors. It is well known that root cause analysis is one of the most important tools we have in the medical system to evaluate errors. This approach allows determination of the scope of the problem, isolation of system failures and design of interventions for improvement.

While our sample size was small and incidence of HGEs in our hospital was low $(11 \%)$, we were able to significantly reduce HGE time to recovery and follow-up. We recognise that this was a single-site, local study without a randomised control design. However, it serves as an important step and warrants testing in other settings with a randomised design.

The biggest challenge we faced in this study included coordinating the nutrition department changes with the bundle-of-care initiative. Ideally, in patients who are able to tolerate oral intake, we recommend they order a snack or meal immediately after the HGE is recognised to prevent further events after treatment administration. ${ }^{14}$

\section{CONCLUSION}

We believe our initiative reflects a cost-effective, patient-centred approach to improving management response to critical hypoglycaemia. Our study illustrates the impact of system-based changes with appropriate standardised protocols to guarantee delivery of care to the right patient at the right time. The proposed hypoglycaemia bundle has been extended to our entire hospital, and we believe it can be applied to other hospitals aiming to improve quality of care and patient safety. Future studies are needed to further document challenges in other specialised units, including labor and delivery or critical care units.

Acknowledgements The authors thank Dr Kasia Lipska of Endocrinology at the Yale School of Medicine, who provided guidance in the determination of primary outcomes and editing feedback and Dr Jenny Blau From the Eunice Kennedy Shriver National Institutes of Child Health and Human Development (NICHD) who provided drafting feedback. The authors also thank the Bridgeport Hospital Internal Medicine Department; Lynda Cook, Clinical Redesign Project; Karri Davis RN, all the diabetes nurse educators, Yale New Haven Health System; Peg Parniawski, RN, Yale New Haven Health System Bridgeport Hospital; and Dr Silvio Inzucchi, Endocrinology, Yale School of Medicine.

Contributors KAA, DKK, GB and CAF had full access to all of the data in the study and take responsibility for the integrity of the data and the accuracy of the data analysis. Study concept and design: KAA, GB, CAF. Acquisition, analysis or interpretation of data: KAA, DKK, NG, GB, CAF. Drafting of the manuscript: KAA, $\mathrm{GB}, \mathrm{CAF}$. Critical revision of the manuscript for important intellectual content: All authors.Statistical analysis: KAA, DKK, CAF, GB. Obtained funding: Bridgeport Hospital Internal Medicine Department. Administrative, technical or material support: Bridgeport Hospital. Study supervision: CAF.

Funding Yale New Haven Health Bridgeport Hospital had no role in the design and conduct of the study; collection, management, analysis, and interpretation of the data. All authors re viewed the manuscript prior to submission. The funding organisations otherwise had no role in the preparation and decision to submit the manuscript for publication.

Competing interests None declared.

Provenance and peer review Not commissioned; externally peer reviewed.

Open Access This is an Open Access article distributed in accordance with the Creative Commons Attribution Non Commercial (CC BY-NC 4.0) license, which permits others to distribute, remix, adapt, build upon this work non-commercially, and license their derivative works on different terms, provided the original work is properly cited and the use is non-commercial. See: http://creativecommons.org/ licenses/by-nc/4.0/

(C) Published by the BMJ Publishing Group Limited. For permission to use (where not already granted under a licence) please go to http://www.bmj.com/company/ products-services/rights-and-licensing/

\section{REFERENCES}

1. The Diabetes Control and Complications Trial Research Group. The effect of intensive treatment of diabetes on the development and progression of long- term complications in insulin-dependent diabetes mellitus. N Engl J Med 1993;329:977-86.

2. Lazar HL, Chipkin SR, Fitzgerald CA, et al. Tight glycemic control in diabetic coronary artery bypass graft patients improves perioperative outcomes and decreases recurrent ischemic events. Circulation 2004;109:1497-502.

3. Nathan DM, Cleary PA, Backlund JY, et al. Intensive diabetes treatment and cardiovascular disease in patients with type 1 diabetes. N Engl J Med 2005;353:2643-53. 
4. Kelly TN, Bazzano LA, Fonseca VA, et al. Systematic review: glucose control and cardiovascular disease in type 2 diabetes. Ann Intern Med 2009;151:394-403.

5. Bonds DE, Miller ME, Bergenstal RM, et al. The association between symptomatic, severe hypoglycaemia and mortality in type 2 diabetes: retrospective epidemiological analysis of the ACCORD study. BMJ 2010;340:b4909.

6. Adler GK, Bonyhay I, Failing H, et al. Antecedent hypoglycemia impairs autonomic cardiovascular function: implications for rigorous glycemic control. Diabetes 2009;58:360-6.

7. Carey M, Boucai L, Zonszein J. Impact of hypoglycemia in hospitalized patients. Curr Diab Rep 2013;13:107-13.

8. Brutsaert E, Carey M, Zonszein J. The clinical impact of inpatient hypoglycemia. J Diabetes Complications 2014;28:565-72.

9. Rajendran R, Rayman G. Serious harm from inpatient hypoglycaemia: a survey of hospitals in the UK. Diabet Med 2014;31:1218-21.

10. Turchin A, Matheny ME, Shubina M, et al. Hypoglycemia and clinical outcomes in patients with diabetes hospitalized in the general ward. Diabetes Care 2009;32:1153-7.

11. Curkendall SM, Natoli JL, Alexander CM, et al. Economic and clinical impact of inpatient diabetic hypoglycemia. Endocr Pract 2009;15:302-12.

12. Auer RN. Hypoglycemic brain damage. Metab Brain Dis 2004;19:169-75.

13. Kerr D, MacDonald IA, Tattersall RB. Influence of duration of hypoglycemia on the hormonal counterregulatory response in normal subjects. J Clin Endocrinol Metab 1989;68:1118-22.
14. Standards of Medical Care in Diabetes-2016: Summary of Revisions. Diabetes Care 2016;39(Suppl 1):S4-5.

15. American Diabetes Association. Standards of medical care in diabetes-2015 abridged for primary care providers. Clin Diabetes 2015;33:97-111.

16. Boord JB, Greevy RA, Braithwaite SS, et al. Evaluation of hospital glycemic control at US academic medical centers. J Hosp Med 2009;4:35-44.

17. Maynard G, Kulasa K, Ramos P, et al. Impact of a hypoglycemia reduction bundle and a systems approach to inpatient glycemic management. Endocr Pract 2015;21:355-67.

18. Noschese M, Donihi AC, Koerbel G, et al. Effect of a diabetes order set on glycaemic management and control in the hospital. Qual Saf Health Care 2008;17:464-8.

19. Schnipper JL, Liang CL, Ndumele CD, et al. Effects of a computerized order set on the inpatient management of hyperglycemia: a cluster-randomized controlled trial. Endocr Pract 2010;16:209-18.

20. Wexler DJ, Shrader P, Burns SM, et al. Effectiveness of a computerized insulin order template in general medical inpatients with type 2 diabetes: a cluster randomized trial. Diabetes Care 2010;33:2181-3.

21. Summary, Evidence -Based Medicine and Changing Nature of Healthcare: 2007 IOM Annual Meeting Summary. Washington (DC), 2008. 